\title{
Can exocentric direction be dissociated from its exocentric distance in virtual environments?
}

\author{
J.A. Aznar-Casanova \\ University of Barcelona, Barcelona, Spain \\ E. H. Matsushima \\ Universidade Federal Fluminense, Niterói, Brasil \\ J.A. DA SILVA \\ Universidade de São Paulo at Ribeirão Preto, Brasil \\ AND \\ N. P. RIBEIRO-FILHO \\ Universidade Federal do Rio de Janeiro, Rio de Janeiro, Brasil
}

\begin{abstract}
We conducted two psychophysical experiments to investigate the relationship between processing mechanisms for exocentric distance and direction. In the first experiment, the task was to discriminate exocentric distances. In the second one, the task was to discriminate exocentric directions. The individual effects of distance and direction on each task were dissociated by analyzing their corresponding psychophysical functions. Under stereoscopicviewing conditions, distance judgments of exocentric intervals were not affected by exocentric direction. However, direction judgments were influenced by the distance between the pair of stimuli. Therefore, the mechanism processing exocentric direction is dependent on exocentric distance, but the mechanism processing exocentric distance does not require exocentric direction measures. As a result, we suggest that exocentric distance and direction are hierarchically processed, with distance preceding direction. Alternatively, and more probably, a necessary condition for processing the exocentric direction between two stimuli may be to know the location of each of them.
\end{abstract}

Most studies that investigate the metrics of visual space use tasks in which observers need to compare or estimate distances, visual directions, angles, slants, areas, and so forth (e.g., Da Silva, 1985; Foley, Ribeiro-Filho, \& Da Silva, 2004; Fukusima, Loomis, \& Da Silva, 1997; Gilinsky, 1951; Gogel, 1961, 1977; Koenderink, van Doorn, \& Lappin, 2000; Loomis, Da Silva, Fujita, \& Fukusima, 1992; Matsushima, de Oliveira, Ribeiro-Filho, \& Da Silva, 2005; Toye, 1986; Wagner, 1985; Wolfe, Maloney, \& Tam, 2005). A commonly observed result is differences in the accuracy of egocentric and exocentric distance judgments (Howard \& Rogers, 2002; Indow, 2004; Wagner, 2006; for a review, see Sedgwick, 1986).

Another common finding is that the visual angle subtended between the visual lines connecting two objects is a robust predictor of the estimated distance (Levin \& Haber, 1993; Matsushima et al., 2005). The results of both studies indicate that the greater the visual angle, the larger the overshooting of the corresponding distance between these objects, which will henceforth be referred to as the exocentric distance. Therefore, visual angle is tightly correlated with exocentric distance (the distance between two objects). Complementarily, an imaginary line connecting the two objects may be considered as an angle relative to an axis of a given coordinate system. Henceforth, this angle will be referred to as exocentric direction.

These two variables, exocentric distance and exocentric direction, are elements in a frame of reference used by observers to organize their visual space. In an egocentric frame of reference, the origin of the coordinate system is the observer. The tasks described here were performed in an exocentric frame of reference, in which spatial locations were defined in relation to external landmarks (Matsushima \& Ribeiro-Filho, 2003), including their distance and direction relative to the origin of the frame of reference. In an exocentric frame of reference, distances and directions are defined relative to an external physical pivot. One must determine the relation between perceived distances and directionsthat is, the intrinsic geometry of the exocentric frame of reference. In addition, the relation that distances and directions have with the physical geometry - that is, the psychophysics of this frame of reference - has to be determined.

Many investigations (Foley, 1968; Künnapas, 1968; Philbeck \& Loomis, 1997; see Sedgwick, 1986, for a review) have been devoted to studying the component of the visual system that operates in an egocentric frame of

J.A.Aznar-Casanova, jaznar2@ub.edu 
reference, in which egocentric distances and egocentric visual directions are vector components of a polar coordinate system. Such a frame of reference generates bodycentered representations, also known as view-dependent representations. However, a visuospatial perception system must also operate with an exocentric frame of reference, which encodes exocentric distances and directions as its vector components and generates environmentcentered, or view-independent, representations. Exocentric distance and direction could be considered as complementary elements that, together, compose the perceptual representation of the environment. To fully understand how this exocentric frame of reference is organized, one must determine how exocentric distance and exocentric direction are related: whether they operate independently or are related and constrain one another in their perceptual properties. Some evidence for interdependency between exocentric distance and direction has been found in studies with spatial anisotropies. These studies showed that the anisotropies in visual space were a consequence of the effects of the direction or orientation of spatial intervals on distance perception (Levin \& Haber, 1993; Loomis \& Philbeck, 1999; Matsushima, 2003; Matsushima et al., 2005). In addition, these studies showed that the visual angle (or exocentric direction) had some effects on the accuracy of exocentric distance estimates. The greater the visual angle, the larger the overshooting of distance estimates, even when the observer's distance from the layout varied and different viewing conditions were used (Matsushima, 2003; Matsushima et al., 2005). Thus, the specific mechanisms of exocentric direction processing may influence the processing of exocentric distance. Alternatively, exocentric distance estimates and exocentric direction estimates could affect one another; that is, there may be reciprocal interactions between them. The dependency between these estimates could be asymmetrical. If this were the case, it would involve hierarchical processing, which requires, for example, previous processing of distance to operate with direction or vice versa.

\section{Overview of the Experiments}

The aim of the present study was to verify whether judgments of an exocentric distance were dissociable from its exocentric direction. Two psychophysical experiments were performed in virtual space. Experiment 1 focused observers on distances, and Experiment 2 focused participants on direction. In Experiment 1, the participants discriminated between exocentric distances. In Experiment 2, the participants discriminated between exocentric directions. These two tasks consisted of two-alternative forced choice tasks. The psychophysical measure was the point of subjective equality (PSE), taken from the plot of the probability of a comparison stimuli larger than standard stimuli response $(C p>S t)$.

In order to dissociate the exocentric distance and direction influences from each other, we combined exocentric distance and direction in the stimuli but dissociated them in the analyses. In Experiment 1, the participants judged whether the exocentric distances of comparison stimuli were longer than those of standard stimuli, for every com- bination of nine distances and nine directions (the standard stimulus was always the same: at the same distance and in the same direction). In Experiment 2, the participants judged whether the comparison stimuli for exocentric direction were greater than the standard stimuli, again for every combination of directions and distances.

To dissociate exocentric distance and direction influences, analyses focused either on exocentric distances, collapsing across exocentric directions, or on exocentric directions, collapsing across exocentric distances. As is depicted in Figure 1 (top left panel), independent analyses of exocentric distances could be extracted by averaging across columns, considering every direction as a repetition of the distance judgment on a constant-stimuli psychophysical task. The averages of the proportion of $C p>S t$ as a function of exocentric distances would fit to a cumulative Gaussian function (Figure 1, lower panel). Analyses of the exocentric direction influence on exocentric distance judgments could be conducted by averaging across rows (Figure 1, top left panel), considering distances as repetitions in a constant-stimuli experiment. Now, the proportion of $C p>S t$ as a function of exocentric direction would fit to a linear function (Figure 1, right panel). A slope equal to zero would indicate that exocentric direction had no effect on exocentric distance perception. In contrast, if the slope was different from zero, the exocentric direction of the interval affected judgments of its exocentric distance.

The dissociation analyses in Experiment 2 are depicted in Figure 2. Analyses of exocentric direction can be extracted by averaging across rows (Figure 2, top left panel), considering distances as repetitions of direction judgments and the proportion of $C p>S t$ as a function of exocentric directions that would fit to a cumulative Gaussian function (Figure 2, right panel). Analyses of exocentric distance effects on exocentric direction judgments can be found by averaging across columns (Figure 2, top left panel), considering directions as repetitions and the proportion of $C p>S t$ as a function of exocentric distance that would fit to a linear function (Figure 2, lower panel). If the slope is different from zero, exocentric distance affects direction processing. If it is equal to zero, there is no influence.

\section{EXPERIMENT 1 Distance Comparisons}

\section{Method}

Participants. The participants included 3 male volunteers (from 32 to 38 years of age) who were experienced in both stereoscopic viewing and psychophysical experiments. All of them had normal or corrected-to-normal vision and were naive as to the experimental objectives and predictions.

Stimuli and Apparatus. A virtual space was generated by a well-known graphic library called OpenGL (Silicon Graphics) in $\mathrm{C}++$ language on a Pentium IV $3-\mathrm{GHz}$ computer. This software enabled us to control a Wildcat VP880 graphic card (3DLabs) connected to LCD stereo shutter goggles (Crystal Eyes) and a 22-in. CRT monitor (Philips Brilliance 202 P4) with 1,024 $\times 768$ pixels of resolution. This ensemble allowed us to generate and display 3-D scenes composed of several pairs of stimuli (red cylinders) against a black background. The stimuli were located at specific egocentric 

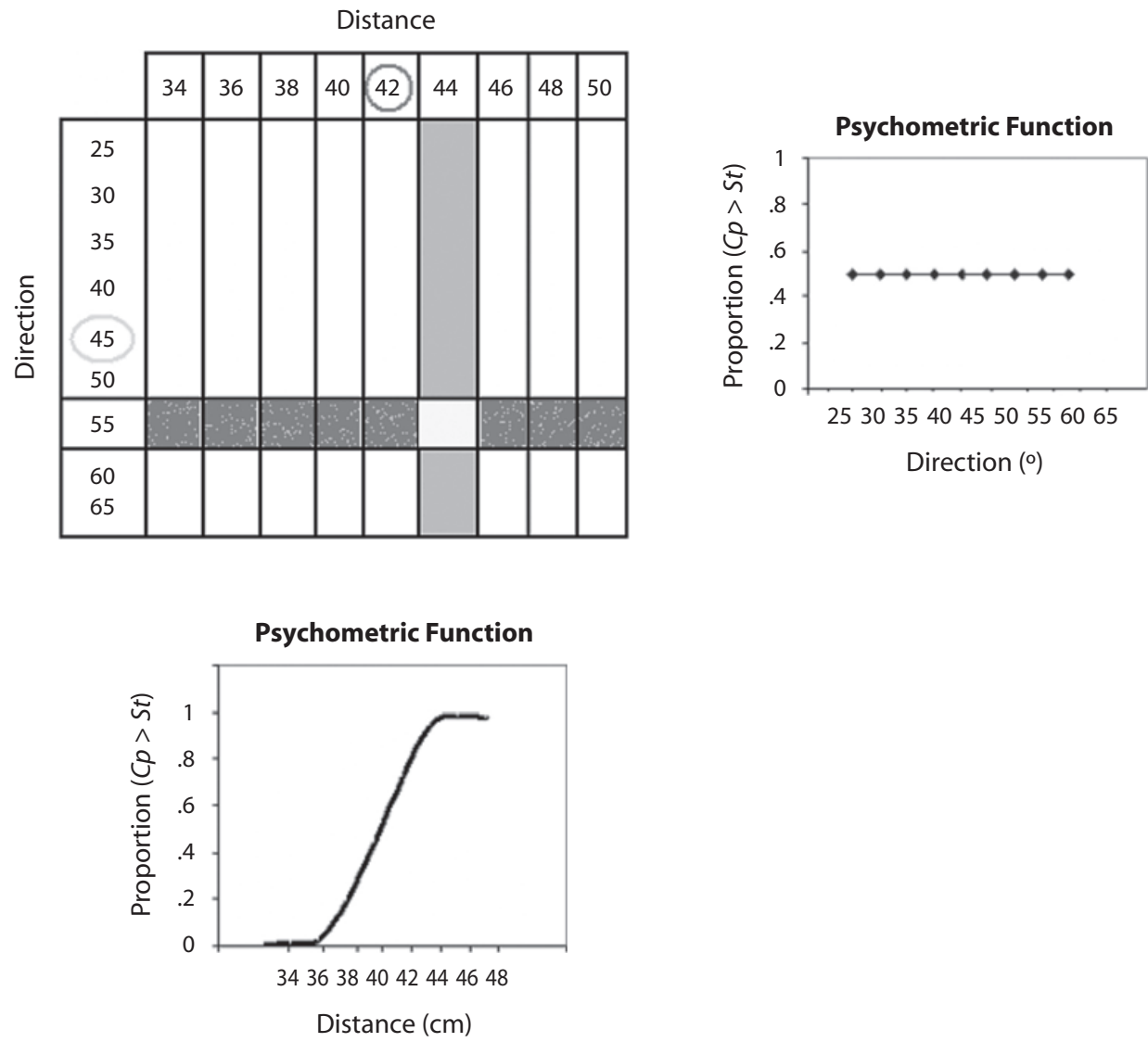

Figure 1. Structure of trials and predictions for the exocentric distance judgment task. The standard stimulus is defined by the circled values of distance and direction variables.

distances and exocentric distances and in different exocentric directions. The luminance of the figure (red cylinder) was $40 \mathrm{~cd} / \mathrm{m}^{2}$. The luminance of the background was $0.008 \mathrm{~cd} / \mathrm{m}^{2}$ (which prevented undesirable aftereffects on the black screen). Since the virtual environment was fully specified by binocular disparity, the size of the stimuli remained constant, and there was no texture gradient; that is, the only available visual source of information was binocular disparity. A chinrest allowed us to keep a 1-m constant viewing distance and prevented head movements.

One of each pair of stimuli (standard and comparison) was always located at the origin of this virtual space at coordinate $0,0,0$, which was located in the leftmost vertex in the screen plane. The other stimulus could be located in front of the screen (near-intervals condition, or crossed disparity) or behind the screen (far-intervals condition, or uncrossed disparity). There were 81 comparison stimuli from the combination of nine different exocentric directions (from $25^{\circ}$ to $60^{\circ}$ in $5^{\circ}$ steps, relative to the screen) and of nine exocentric distances (from 34 to $50 \mathrm{~cm}$ in 2-cm steps). The standard stimulus had a fixed $42-\mathrm{cm}$ exocentric distance and a fixed $45^{\circ}$ exocentric direction. In Figure 3, we show a sketch of two pairs of stimuli (standard stimuli in white and comparison stimuli in gray) for near and far conditions. Each cylinder was $0.40 \mathrm{~cm}$ in diameter $\times 8.00 \mathrm{~cm}$ high. From the viewing distance, the vertical size of the cylinder subtended a visual angle of $4.6^{\circ}$. Therefore, since 1 stimulus was placed in the origin (in front of the observer's main visual line) and the other stimulus determined the exocentric direction, the subtended visual angles could vary from $6.27^{\circ}$ (in the case of a far condition for an exocentric distance of $34 \mathrm{~cm}$ and an exocentric direction of $65^{\circ}$ ) to $29.87^{\circ}$ (in the case of a near condition for an exocentric distance of $50 \mathrm{~cm}$ and an exocentric direction of $25^{\circ}$ ).

Procedure. The participants were positioned $1 \mathrm{~m}$ from the screen and read the instructions, which informed them that two pairs of stimuli would be presented on each trial and that they had to pay attention to the distance between them. The participants' task was to decide whether the exocentric distance of the comparison stimuli was longer than that of the standard stimuli. The task was performed under near and far stereo-viewing conditions, as shown in Figure 3.

After the participants read the instructions, the experiment began with 20 practice trials, followed by three blocks of 81 trials under one of the stereo-viewing conditions (either near or far), with a 5-min resting time between each block. On the following day, the participants engaged in another session, in the other stereo-viewing condition. The order of the stereo-viewing conditions was counterbalanced across participants. Each block took approximately $10 \mathrm{~min}$, since the participants had no time limits for producing their responses.

Each trial followed this sequence: presentation of the standard pair of stimuli for $2 \mathrm{sec}$; $1 \mathrm{sec}$ of black screen; and presentation of the comparison pair for $2 \mathrm{sec}$. The participants pressed the right button of the mouse for $C p>S t$ and the left button for $C p<S t$. Every participant undertook 486 trials $(2$ stereo-viewing conditions $\times 3$ series of trials $\times 9$ distances $\times 9$ directions).

\section{Results and Discussion}

The data and regression fits for far and near distances are depicted in Figures 4 and 5, respectively. We averaged 

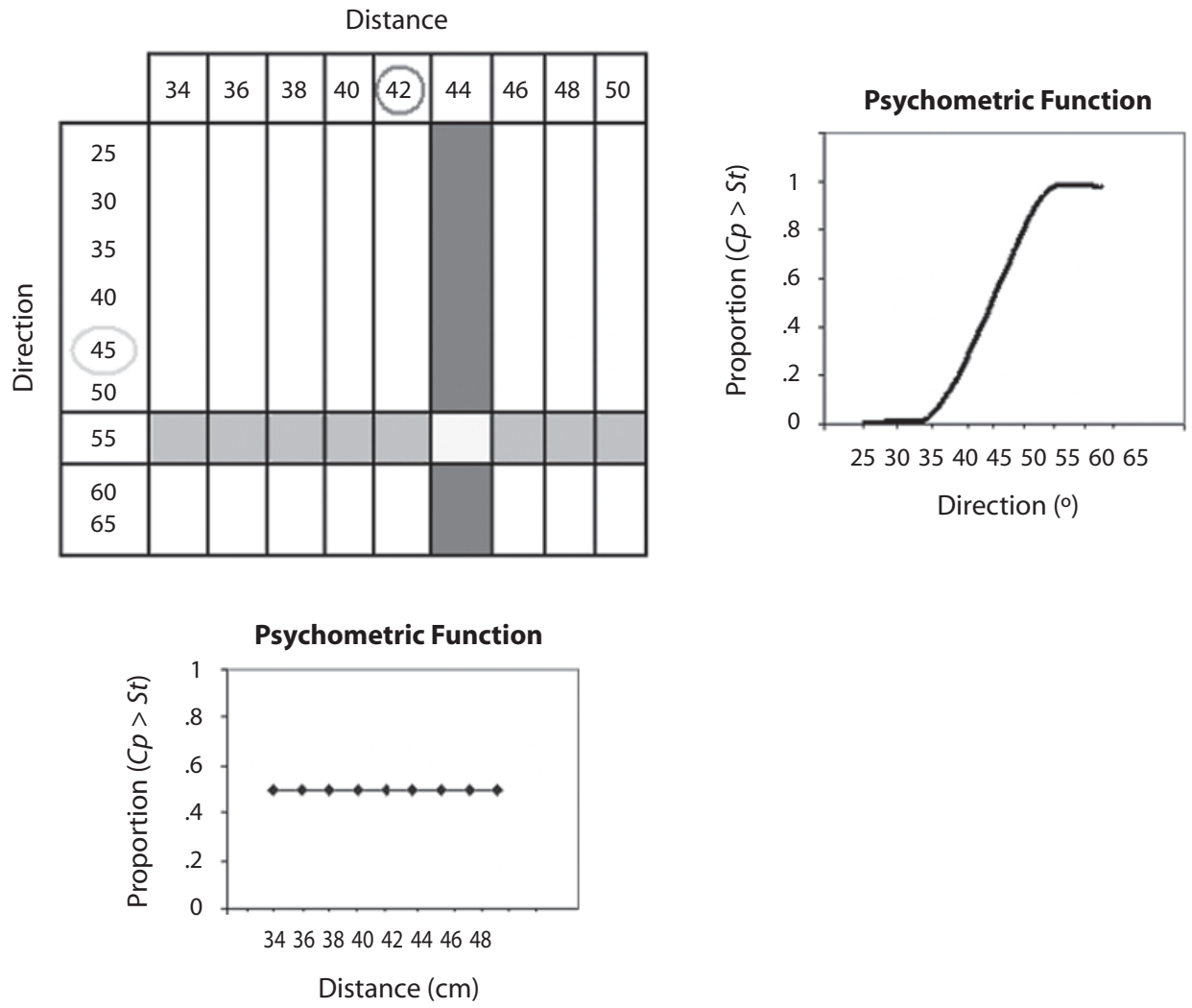

Figure 2. Structure of trials and predictions for the exocentric direction judgment task. The standard stimulus is defined by the circled values of distance and direction variables.
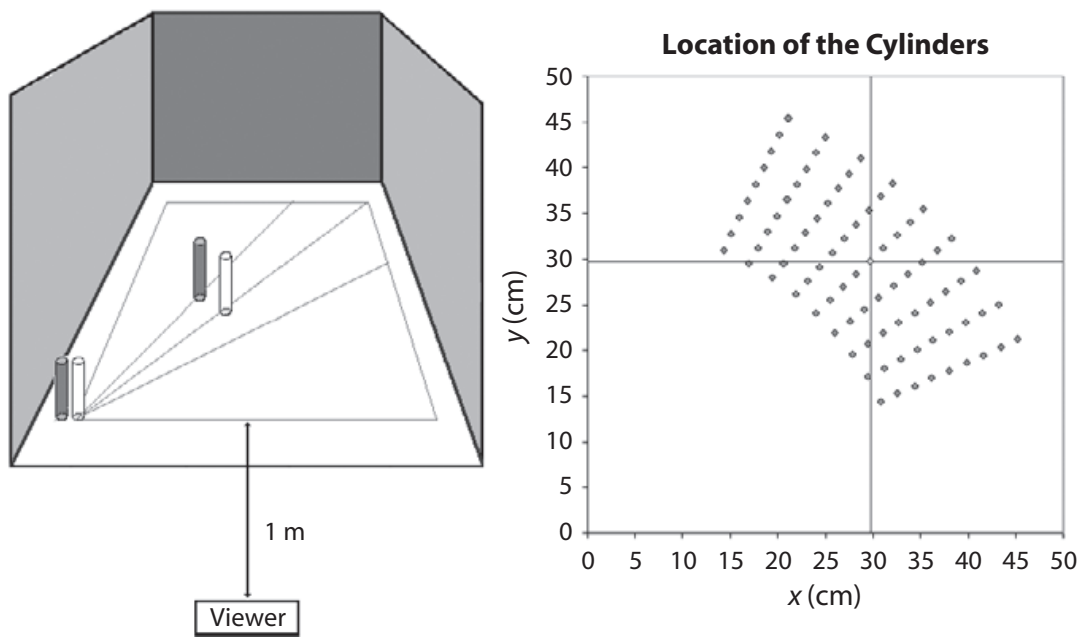

Figure 3. A sketch of the virtual space for uncrossed disparities (far distances, in upper plane) and for crossed disparities (near distances, in lower plane). In distance judgments, the participants compared the extent of comparison stimuli (gray) with the extent of standard stimuli (white). In direction judgments, the participants compared the direction of comparison stimuli (gray) with the direction of standard stimuli (white). Since the location (origin) of one stimulus was the same for the comparison and standard pairs, we placed the gray stimulus above the white one but presented it in the white stimulus position. Note that the observer position was $1 \mathrm{~m}$ from the screen. 
over participants, since they all presented essentially the same pattern of results. An analysis of distance comparisons for far conditions shows that when the participants focused on exocentric distance, the differences between the distance discriminations for standard and comparison stimuli fit a cumulative Gaussian function $\left(R^{2}=.991\right)$, with mean or PSE $=41.581$ (confidence interval = 41.089-42.072) and $S D=4.643$ (confidence interval = 3.930-5.356). The root of the mean squared error (RMSE) is .032. The mean constant error (CE) obtained is -.4194 . The mean differential threshold (DT) is 2.712, and the mean Weber fraction .065.

In contrast, an analysis of the exocentric direction when distances for far conditions were judged revealed that the data fit a linear function, with slope $=-.001(S E=.001)$ and intercept $=.559$. The slope does not differ from zero $(p=.225)$.

A similar pattern of parameters was found for near conditions. The data (proportion of $C p>S t$ as a function of distance) fit a cumulative Gaussian function $\left(R^{2}=.957\right)$, $\mathrm{PSE}=41.929($ confidence interval $=40.914-42.943)$ and $S D=10.477$ (confidence interval $=8.210-12.743)$. The RMSE is .039, and the mean CE is -.071 . The mean DT is 6.995, and the mean Weber fraction .167. However, if we analyze the distance judgments for varying exocentric directions under near conditions, the data fit a linear function with slope $=-.001(S E=.001)$ and intercept $=.559$. Once again, the slope does not differ from zero $(p=.182)$. Figure 5 shows the relationship between these two functions.

Tables 1 and 2 present all the relevant psychophysical parameters for far and near conditions, respectively. Parameters are given as a function of exocentric direction. The PSE closely fluctuates around the standard stimuli $(42 \mathrm{~cm})$. However, the slopes of the cumulative Gaussian function $(S D)$, constant errors, discrimination thresholds, and Weber fractions vary as the exocentric direction deviates from $45^{\circ}$. In general, the data fit a cumulative Gaussian.

These results show that exocentric distance judgments are not affected by the exocentric direction in the range of direction angles examined here $\left(25^{\circ}-65^{\circ}\right.$, in $5^{\circ}$ steps $)$. However, several other studies performed outdoors in an open field (Levin \& Haber, 1993; Matsushima et al., 2005) have shown that visual angle (or slant) affects the exponent of the power law. Explanations for this apparent discrepancy between results are twofold. First, in the present experiment, the dominant visual cue was binocular disparity, whereas in the other studies, full-cue environments provided many depth cues. Second, the range of distances in the present investigation was small and restricted to peripersonal space. In contrast, the range of distances in the other studies was large and involved the action space.

\section{EXPERIMENT 2 Direction Comparisons}

\section{Method}

Participants. The sample in Experiment 1 also participated in Experiment 2.

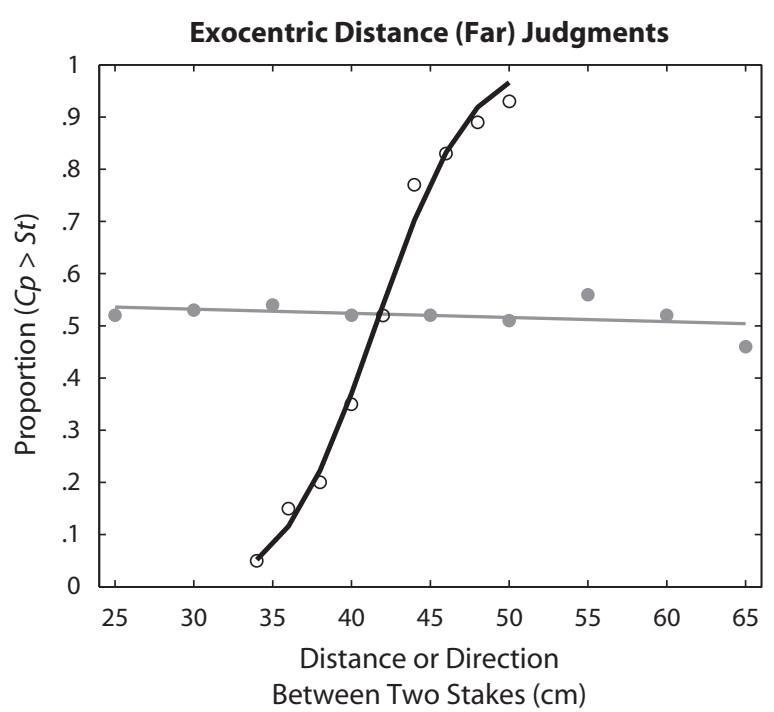

Figure 4. Proportion of $C p>S t$ as a function of exocentric distance and direction for the distance judgments, for far distances (uncrossed disparities). The thick line and open circles represent the distance judgments. The thin line and filled circles represent the direction analysis of the same data.

Stimuli and Apparatus. The experimental setting was exactly the same as that in Experiment 1.

Procedure. The same procedure as that in Experiment 1 was used in Experiment 2, except that in Experiment 2, the task was to decide whether the exocentric direction of comparison stimuli was greater than that of standard stimuli.

The structure of this test and the experimental sequence was similar, but opposite to, that used in Experiment 1 (as described in the

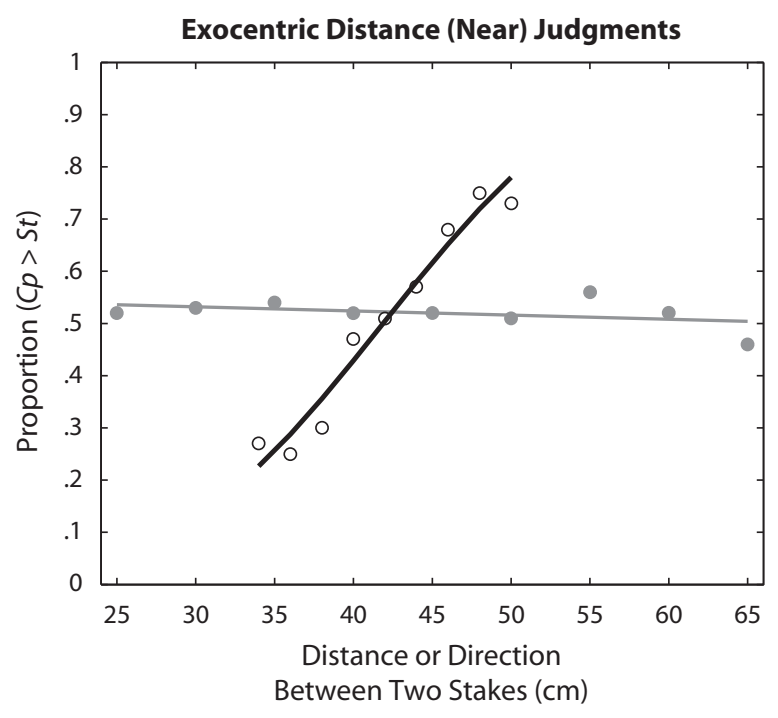

Figure 5. Proportion of $C p>S t$ as a function of exocentric distance and direction for the distance comparisons, for near distances (crossed disparities). The thick line and open circles represent distance judgments. The thin line and filled circles represent the direction analysis of the same data. 
Table 1

Psychophysical Parameters for Exocentric Far Distances As a Function of the Exocentric Direction of Stimuli

\begin{tabular}{cccccccc}
\hline $\begin{array}{c}\text { Direction } \\
\left({ }^{\circ}\right)\end{array}$ & PSE & \multicolumn{1}{c}{$S D$} & RMSE & $R^{2}$ & \multicolumn{1}{c}{ CE } & \multicolumn{1}{c}{ DT } & Weber \\
\hline 25 & 41.547 & 4.267 & .111 & .900 & -0.453 & 2.425 & .058 \\
30 & 41.205 & 1.810 & .080 & .968 & -0.795 & 0.430 & .010 \\
35 & 40.988 & 3.140 & .046 & .987 & -1.012 & 1.100 & .027 \\
40 & 41.910 & 2.690 & .146 & .884 & -0.090 & 1.725 & .041 \\
45 & 42.074 & 1.209 & .048 & .990 & 0.074 & 0.889 & .021 \\
50 & 42.018 & 5.572 & .121 & .864 & 0.018 & 3.777 & .899 \\
55 & 41.051 & 4.382 & .065 & .968 & -0.949 & 2.006 & .049 \\
60 & 41.297 & 11.214 & .136 & .626 & -0.703 & 6.861 & .166 \\
65 & 43.536 & 13.182 & .097 & .701 & 1.536 & 10.427 & .240 \\
\hline
\end{tabular}

Note-PSE, point of subjective equality; $S D$, standard deviation; RMSE, root of the mean squared error; CE, mean constant error; DT, differential threshold.

Overview of the Experiments section). The task, the trial sequence for each test, and the response mode were similar to those in the previous experiment. As a result, the participants undertook a total of 486 trials.

Given that the participants in Experiment 2 were the same as those in Experiment 1, we partially counterbalanced the order of the trials and experiments to prevent learning effects. For instance, if Participant 1 performed the Trial 1, Experiment 1-Trial 1, Experiment 2-Trial 2, Experiment 1-Trial 2, Experiment 2 sequence, Participant 2 would have performed the reverse Trial 2, Experiment 2-Trial 2, Experiment 1-Trial 1, Experiment 2-Trial 2, Experiment 1 sequence, and Participant 3 would have performed another, different sequence.

\section{Results and Discussion}

Data and regression fits for far and near conditions are depicted in Figures 6 and 7, respectively. The data (the proportion of $C p>S t$ as a function of direction) for direction judgments under far conditions fit a cumulative Gaussian function $\left(R^{2}=.989\right)$, with PSE $=45.227$ (confidence interval $=43.868-46.587$ ) and $S D=10.312$ (confidence interval $=8.371-12.253$ ). The RMSE is .038 , the mean CE is .227 , the mean DT is 7.183 , and the mean Weber fraction is .159. In contrast, when the participants focused on distance while judging exocentric directions, the resulting data produced fit a linear function $\left(R^{2}=.980\right)$, whose intercept is 1.545 and slope is $-.025(S E=.005)$. Nevertheless, this slope significantly differs from zero $(F=132.900, p=.0001)$.

Table 2

Psychophysical Parameters for Exocentric Near Distances As a Function of the Exocentric Direction of Stimuli

\begin{tabular}{ccrccccc}
\hline $\begin{array}{c}\text { Direction } \\
\left({ }^{\circ}\right)\end{array}$ & PSE & \multicolumn{1}{c}{$S D$} & RMSE & $R^{2}$ & CE & DT & Weber \\
\hline 25 & 43.92 & 9.58 & .089 & .825 & 1.92 & 8.38 & .191 \\
30 & 42.07 & 10.11 & .080 & .847 & 0.70 & 6.89 & .164 \\
35 & 41.87 & 10.11 & .095 & .800 & -0.12 & 6.70 & .159 \\
40 & 41.48 & 10.40 & .088 & .810 & -0.090 & 6.50 & .157 \\
45 & 42.01 & 10.33 & .089 & .810 & -0.013 & 6.98 & .166 \\
50 & 42.07 & 9.39 & .081 & .860 & 0.067 & 6.40 & .152 \\
55 & 39.86 & 9.06 & .090 & .840 & -2.14 & 3.97 & .100 \\
60 & 41.90 & 10.63 & .111 & .720 & -0.10 & 7.07 & .169 \\
65 & 42.31 & 16.08 & .064 & .800 & 0.31 & 11.15 & .264 \\
\hline
\end{tabular}

Note-PSE, point of subjective equality; $S D$, standard deviation; RMSE, root of the mean squared error; CE, mean constant error; DT, differential threshold.
A similar pattern was found for near conditions. Direction comparisons fit a cumulative Gaussian function $\left(R^{2}=.995\right), \mathrm{PSE}=45.921$ (confidence interval $=$ 45.041-46.800) and $S D=8.728$ (confidence interval $=$ 7.482-9.974). The RMSE is .026, the mean CE is .921, the mean DT is 6.808, and the mean Weber fraction is .148. When the participants analyzed distances while judging exocentric directions, the data fit a linear function $\left(R^{2}=\right.$ .970 ), whose intercept is 1.157 and slope is -.016 . ( $S E=$ $.0001)$. However, this slope also reliably differs from zero $(F=329.100, p=.0001)$.

Table 3 summarizes the most relevant psychophysical parameters for far conditions as a function of exocentric distances. The PSE varies increasingly with distance. In addition, the accuracy of judgments $(S D)$ also varies accordingly to distance. $\mathrm{CE}$ increases as comparison stimuli deviate from the standard value $(42 \mathrm{~cm})$. This pattern is also observed in DT and Weber fractions.

A comparison of psychophysical parameters under far- and near-interval conditions (see Tables 3 and 4) shows that the main difference occurs in constant errors. An asymmetry between crossed and uncrossed disparity has been found. However, this finding is not unequivocal. Our findings agree with the results of some previous investigations. Westheimer and Tanzman (1956) revealed that observers detected the location of a stimulus in uncrossed disparity more reliably than in crossed disparity. Their study used briefly presented stereoscopic stimuli, containing large disparities (between $1^{\circ}$ and $10^{\circ}$ ). Observers in Schor and Wood's (1983) experiment for lowspatial-frequency stereograms (DOG patches) perceived less depth in crossed disparity than in uncrossed disparity. The results of other studies have been conflicting (e.g., Lasley, 1985; Schumer \& Julesz, 1984; Woo \& Sillanpaa, 1979), although they have demonstrated the existence of asymmetries (see Mustillo, 1985, for a review on the differences between crossed and uncrossed disparity).

These results show that observers achieve higher levels of accuracy in exocentric direction judgments as the stimuli get more separated. Therefore, the exocentric distance of the interval affects exocentric direction judgments. A minimum distance between objects may be needed to accurately discriminate exocentric direction.

\section{GENERAL DISCUSSION}

We conducted two experiments to investigate the relationship between the processing of exocentric distances and directions. In Experiment 1, we asked the participants to make discriminative judgments of exocentric distances between two stimuli while varying their direction. In Experiment 2, we asked the participants to judge the exocentric directions between two stimuli while varying their distance. Therefore, both experiments used the same set of stimuli. However, they differed in the judgment that the participants were asked to make (distance in Experiment 1 and direction in Experiment 2). Subsequently, in Experiment 1, we obtained the psychophysical functions for distance judgments made when exocentric directions were varied (row analysis in Figure 1). In Experiment 2, 


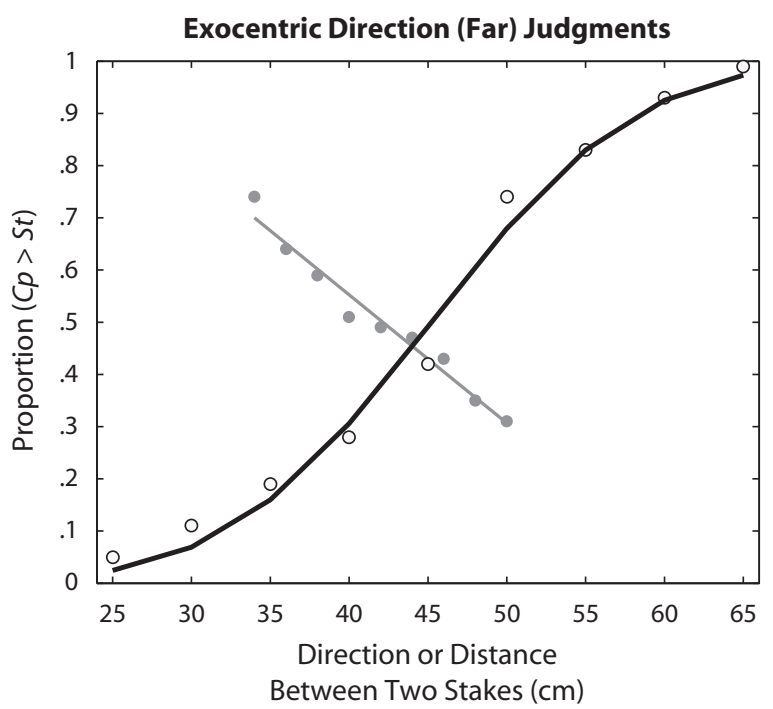

Figure 6. Proportion of $C p>S t$ as a function of exocentric distance and direction for direction judgments, for far directions (uncrossed disparities). The thick line and open circles represent direction judgments. The thin line and filled circles represent the distance analysis of the same data.

we obtained the psychophysical functions for direction judgments and judgments for an incidental task (direction judgments when exocentric distances were varied) implicit in the participants' responses (columns analysis in Figure 2). In this way, we dissociated exocentric distance and direction influences on processing. By examining the corresponding psychophysical functions that fit the data in the incidental task, we could verify whether

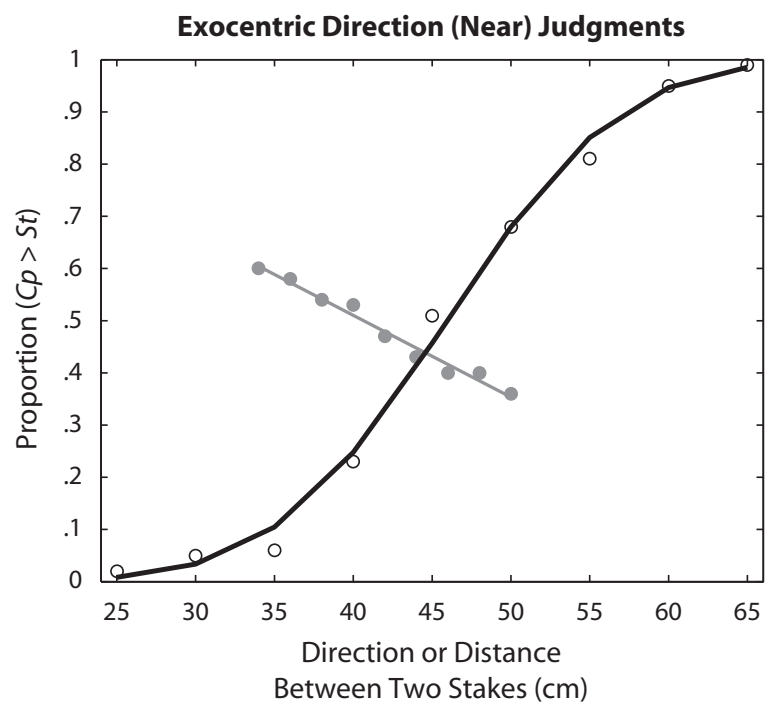

Figure 7. Proportion of $\boldsymbol{C p}>\boldsymbol{S t}$ as a function of exocentric distance and direction for direction judgments, for near directions (crossed disparities). The thick line and open circles represent direction judgments. The thin line and filled circles represent the distance analysis of the same data. these dimensions of visual space (distance and direction) are independent of or dependent on each other.

Our data show that, at least for the virtual scenes specified by stereoscopic information, exocentric distance judgments were not influenced by the exocentric direction subtended by the stimuli. However, exocentric direction judgments were significantly biased as a function of the exocentric distance comprised by the stimuli. This finding suggests that there is dissociation between the mechanisms specialized in distance processing and those specialized in direction processing in an exocentric frame of reference. The results may indicate that exocentric direction processing follows processing of exocentric distance.

However, considering the specific layout structure of our displays, this dependency of exocentric distance on the processing of exocentric direction may not be the only explanation for the results. Since one of the stimuli in every pair (standard and comparison pairs of stimuli) was always located at the origin, the participants could simply make their exocentric direction discriminations on the basis of whether the second stimulus of the comparison pair was to the left or the right of the second stimulus of the standard pair. Although this is a plausible strategy, our data ruled out this hypothesis. If the participants used such a strategy, the data summarized in the cumulative Gaussian (e.g., for far distances) would have a pattern different from that presented in Figure 6. The pattern would have been a step function with a cutoff separating two levels: one on the left, which would always be less than the standard, and another on the right, which would always be greater than the standard. Instead, a smooth, cumulative Gaussian was produced.

Previous evidence supports this sequence of processing. It has been proposed (e.g., Foley, 1980; Heller, 2004) that the mechanism for processing egocentric distance is, essentially, the vergence cue (ocular convergence), whereas the mechanism for processing size (or exocentric distance) is the horizontal binocular disparity cue, which basically requires horizontal disparities $(\nabla \mathrm{h})$. In addition, any hypothetical mechanism for processing exocentric directions (orientation of a spatial interval) must integrate three different pieces of evidence.

First, physiological evidence shows that cells with elongated receptive fields tuned to orientation disparity can detect the orientation of a spatial interval. Blakemore, Fiorentini, and Maffei (1972) found evidence for cells' being tuned to orientation disparity. They achieved this by measuring responses in single binocular cells in the cat cortex for a set of bars varying in their orientations. These binocular orientation detectors were more broadly tuned than the monocular ones (bandwidth about $15^{\circ}$, $S D=6^{\circ}$ ). The orientation disparity function of these cells can be derived from the difference between the monocular orientation functions. The visual angle subtended by the spatial interval subtended by the two stimuli decreases as its orientation increases relative to the frontoparallel plane, according to an inverse relation to $\cos (\theta)$, with $\theta$ as the exocentric direction. Such monocular differences due to binocular parallax could probably 
Table 3

Psychophysical Parameters for Exocentric Far Directions As a Function of the Exocentric Distances of Stimuli

\begin{tabular}{ccrcccrr}
\hline $\begin{array}{c}\text { Distance } \\
(\mathrm{cm})\end{array}$ & PSE & \multicolumn{1}{c}{ SD } & RMSE & $R^{2}$ & \multicolumn{1}{c}{ CE } & \multicolumn{1}{c}{ DT } & Weber \\
\hline 34 & 32.459 & 14.393 & .095 & .859 & -12.541 & -2.833 & -.087 \\
36 & 38.192 & 9.742 & .066 & .964 & -6.808 & -0.237 & -.006 \\
38 & 41.202 & 3.944 & .058 & .986 & -3.798 & -1.138 & -.028 \\
40 & 46.459 & 9.380 & .156 & .845 & 1.459 & 7.786 & .168 \\
42 & 44.078 & 6.628 & .065 & .979 & -0.922 & 3.549 & .081 \\
44 & 46.638 & 4.473 & .019 & .998 & 1.638 & 4.655 & .100 \\
46 & 48.941 & 8.019 & .093 & .942 & 3.941 & 9.350 & .191 \\
48 & 53.115 & 6.147 & .104 & .932 & 8.115 & 12.261 & .231 \\
50 & 54.364 & 9.686 & .061 & .967 & 9.364 & 15.897 & .292 \\
\hline
\end{tabular}

Note-PSE, point of subjective equality; $S D$, standard deviation; RMSE, root of the mean squared error; CE, mean constant error; DT, differential threshold.

be used to detect the differential orientation disparities produced by lines slanted in depth about a vertical axis. In summary, differences between the monocular orientations for left and right eyes would specify the orientation of the segment. Our displays could provide this disparity information. However, these physiological features cannot explain the asymmetrical relation between exocentric distance and direction.

The second piece of evidence showing the dissociation between exocentric distance and exocentric direction processing comes from a geometric approach. Indeed, according to the rules for projective transformation, a spatial interval, located at a certain distance from an observer along his/her line of sight, projects an image onto the retina subtending a visual angle $\alpha$. The perception of its orientation depends on two factors. One factor is the viewing distance (egocentric distance), which affects the visual angle and scales the size of the spatial interval. The other factor is the eccentricity $(\varepsilon)$, which decreases the orientation in proportion to $\cos ^{2}(\varepsilon)$. In addition, the retinal image of a horizontal spatial interval in the frontoparallel plane diminishes in a ratio of about $1 / d$ (where $d$ is egocentric distance), whereas the same spatial interval receding in the depth (saggital) plane diminishes in a ratio of about $1 / d^{2}$. As a consequence, when one observes a spatial interval with fixed length, changes in the size and eccen-

Table 4

Psychophysical Parameters for Exocentric Near Directions As a Function of the Exocentric Distances of Stimuli

\begin{tabular}{cccccccc}
\hline $\begin{array}{c}\text { Distance } \\
(\mathrm{cm})\end{array}$ & PSE & $S D$ & RMSE & $R^{2}$ & CE & DT & Weber \\
\hline 34 & 40.164 & 8.279 & .095 & .934 & -4.836 & 0.749 & .019 \\
36 & 40.669 & 6.724 & .106 & .933 & -4.330 & 0.205 & .005 \\
38 & 44.317 & 3.495 & .091 & .969 & -0.682 & 1.675 & .038 \\
40 & 42.585 & 3.532 & .0494 & .996 & -2.415 & 0.032 & .0006 \\
42 & 45.698 & 7.711 & .0517 & .984 & 0.698 & 5.898 & .129 \\
44 & 48.531 & 7.665 & .128 & .905 & 3.531 & 8.701 & .179 \\
46 & 49.756 & 7.893 & .091 & .948 & 7.756 & 10.081 & .203 \\
48 & 51.282 & 8.642 & .094 & .939 & 6.282 & 12.111 & .236 \\
50 & 51.538 & 7.845 & .0580 & .975 & 6.538 & 11.829 & .229 \\
\hline
\end{tabular}

Note-PSE, point of subjective equality; $S D$, standard deviation; RMSE, root of the mean squared error; CE, mean constant error; DT, differential threshold. tricity of the retinal images are converted into changes in perceived orientation. Thus, the monocular sizes of the spatial interval must be the inputs for exocentric orientation processing.

Finally, the third piece of experimental evidence on the processing of orientation comes from psychophysical experiments. With stereoscopic stimuli, it has been demonstrated that horizontal size disparity (HSD) does not specify the size (or exocentric distance). It specifies only the slant of a surface (or line) with respect to the tangent plane of an isodisparity cylinder (Howard \& Rogers, 2002, pp. 222-225). The HSD is a zero-order disparity and can be computed by the differences between the monocular angles subtended between two points, HSD $=\left(\alpha_{1 \mathrm{~L}}-\alpha_{2 \mathrm{~L}}\right)-\left(\alpha_{1 \mathrm{R}}-\alpha_{2 \mathrm{R}}\right)$. It can be influenced by the orientation of the spatial interval, which, in turn, affects its eccentricity and the exocentric distance. However, the horizontal size ratio (HSR) is the ratio of the angular size of the images projected in the left and right eyes:

$$
\operatorname{HSR}=\left(\alpha_{1 \mathrm{~L}}-\alpha_{2 \mathrm{~L}}\right) /\left(\alpha_{1 \mathrm{R}}-\alpha_{2 \mathrm{R}}\right) .
$$

The HSR is a first-order disparity, which provides information about the disparity gradient. The HSR varies inversely with egocentric distance.

Finally, another way of computing the orientation consists of using vertical size disparities, which provide information about eccentricity at a specified distance, as suggested by Rogers and Bradshaw (1994). However, the ratio $\nabla v / \nabla h$ could also be used to predict orientation. If this is true, exocentric direction is a geometrical property that is subsequent to exocentric distance processing, like the relationship between egocentric and exocentric distances.

Together, these results show that there is an asymmetrical relationship between exocentric distance and direction. Exocentric distance judgments were independent of exocentric direction, whereas exocentric distance affected exocentric direction judgments. In summary, our data suggest that the mechanism for exocentric distance processing is dissociable from the mechanism for exocentric direction processing. However, the contrary does not hold. Mechanisms for exocentric direction processing are not independent of the exocentric distance comprised by stimuli. This asymmetry suggests either that the processing is hierarchical, with exocentric distance needing to be processed before exocentric direction can be processed, or, more probably, that a necessary condition for processing the exocentric direction between two stimuli is to know the location of each of them. Another possibility, as has been suggested by Abrams and Landgraf (1990), is that differential use of distance and location information may underlie differences in localization behavior. Perhaps, the difference between the processing of exocentric distances and exocentric directions may be due, at least for stereoscopic presentations, to different uses of these types of spatial information by the perceptual and motor systems.

A tentative explanation for this hierarchical processing was offered by Foley's (1980) binocular distance 
perception model, which is related to the exocentric distance perception model (Foley et al., 2004). Foley (1980) stated that perceived exocentric distance depends on the binocular parallaxes of the targets and is a function of perceived egocentric distance. In order to process exocentric distance, one must extract an egocentric distance signal for each target, converting binocular disparities and binocular parallaxes into effective disparities and parallaxes. Thus, the exocentric distances and directions must be processed as a function of perceived egocentric distances (Foley et al., 2004). Therefore, the processing of exocentric directions is really dependent on distance, although this distance information may be perceived as egocentric distance, which covaries with the simulated exocentric distances.

These results may seem to be in contrast with some previous studies on outdoor environments, which showed lawful interactions between exocentric direction (or visual angle in their concept) and exocentric distance (Levin \& Haber, 1993; Matsushima et al., 2005). These studies showed that exocentric distance judgments were biased by the exocentric direction of the spatial intervals. The accuracy of exocentric distance judgments improved as the exocentric direction increased. However, considering the differences in the methodology of these studies, the different patterns of interactions may be highly correlated. First of all, since all depths in the present study were specified by binocular disparity, this visual cue was dominant in the processing of distances and directions. In the outdoor studies, the distance ranges and the layout of egocentric distances reduced the processing utility of binocular disparity to almost zero. Therefore, other visual cues, such as motion parallax, relative size, or angular declination, must have played relevant roles in the distance and direction processing.

Second, the experimental design of previous studies could not dissociate the exocentric distance and direction contributions from each other in their respective processing. They could indicate only whether a weighting factor was closely related to exocentric direction and modulated the exocentric distance judgments. Since the present investigation provided exocentric distance and direction judgments and its experimental design could dissociate the relative contribution of these judgments, our results tell us about the lawful interactions between exocentric distance and direction with higher levels of internal validity. Previous findings (Levin \& Haber, 1993; Matsushima et al., 2005) do not conflict with the present findings, since their experimental design was not aimed at investigating the dissociation between exocentric distance and direction.

\section{AUTHOR NOTE}

This research was supported by Grant SEJ2006-15095/PSIC from the Spanish Ministry of Science and Technology. We thank the two reviewers assigned to evaluate our article for their valuable comments. We are particularly grateful for an anonymous reviewer's comments, which we have included in the General Discussion. Correspondence concerning this article should be addressed to J. A. Aznar-Casanova, Department of Basic
Psychology, Faculty of Psychology, University of Barcelona, Passeig Vall d'Hebron, 171, 08035 Barcelona, Spain (e-mail: jaznar2@ub.edu).

\section{REFERENCES}

Abrams, R. A., \& Landgraf, J. Z. (1990). Differential use of distance and location information for spatial localization. Perception \& Psychophysics, 47, 349-359.

Blakemore, C., Fiorentini, A., \& Maffei, L. (1972). A second neural mechanism of binocular depth discrimination. Journal of Physiology, 226, 725-749.

DA Silva, J. A. (1985). Scales for perceived egocentric distance in a large open field: Comparison of three psychophysical methods. American Journal of Psychology, 98, 119-144.

Foley, J. M. (1968). Depth, size and distance in stereoscopic vision. Perception \& Psychophysics, 3, 265-274.

Foley, J. M. (1980). Binocular distance perception. Psychological Review, 87, 411-434.

Foley, J. M., Ribeiro-Filho, N. P., \& Da Silva, J. A. (2004). Visual perception of extent and the geometry of visual space. Vision Research, 44, 147-156.

Fukusima, S. S., Loomis, J. M., \& Da Silva, J. A. (1997). Visual perception of egocentric distance as assessed by triangulation. Journal of Experimental Psychology: Human Perception \& Performance, 23, 86-100.

GiLINSKy, A. S. (1951). Perceived size and distance in visual space. Psychological Review, 58, 460-482.

Gogel, W. C. (1961). Convergence as a cue to the perceived distance of objects in a binocular configuration. Journal of Psychology, 52, 303-315.

Gogel, W. C. (1977). The metric of visual space. In W. Epstein (Ed.), Stability and constancy in visual perception: Mechanisms and processes (pp. 129-181). New York: Wiley.

Heller, J. (2004). The locus of perceived equidistance in binocular vision. Perception \& Psychophysics, 66, 1162-1170.

Howard, I. P., \& Rogers, B. J. (2002). Seeing in depth: Vol. 2. Depth perception. Toronto: I Porteus.

InDOw, T. (2004). The global structure of visual space. River Edge, NJ: World Scientific.

Koenderink, J. J., van Doorn, A. J., \& Lappin, J. S. (2000). Direct measurement of the curvature of visual space. Perception, 29, 69-79.

KÜNNAPAS, T. (1968). Distance perception as a function of available visual cues. Journal of Experimental Psychology, 77, 523-529.

LASLEY, D. J. (1985). Discrimination of crossed and uncrossed disparities. Journal of the Optical Society of America A, 2, 399-403.

Levin, C. A., \& Haber, R. N. (1993). Visual angle as a determinant of perceived interobject distance. Perception \& Psychophysics, 54, 250-259.

Loomis, J. M., Da Silva, J. A., Fujita, N., \& Fukusima, S. S. (1992). Visual space perception and visually directed action. Journal of Experimental Psychology: Human Perception \& Performance, 18, 906-921.

Loomis, J. M., \& PHiLbeck, J. W. (1999). Is the anisotropy of perceived 3-D shape invariant across scale? Perception \& Psychophysics, 61, 397-402.

Matsushima, E. H. (2003). Anisotropia do espaço percebido e paralaxe de movimento: Uma análise da orientação espacial (Anisotropy of perceived space and motion parallax: An analysis of spatial orientation). Unpublished doctoral thesis, Universidade de São Paulo, Ribeirão Preto, Brasil.

Matsushima, E. H., de Oliveira, A. P., Ribeiro-Filho, N. P., \& Da SiLva, J. A. (2005). Visual angle as determinant factor for relative distance perception. Psicológica, 26, 97-104.

Matsushima, E. H., \& Ribeiro-Filho, N. P. (2003). Interações entre sistemas de referência alocêntricos e egocêntricos: Evidências dos estudos com direção percebida (Interactions between allocentric and egocentric frames of reference: Evidences from studies with perceived direction). Estudos e Pesquisas em Psicologia, 3, 105-118.

Mustillo, P. (1985). Binocular mechanisms mediating crossed and uncrossed stereopsis. Psychological Bulletin, 97, 187-201. 
Philbeck, J. W., \& Loomis, J. M. (1997). Comparison of two indicators of perceived egocentric distance under full-cue and reduced-cue conditions. Journal of Experimental Psychology: Human Perception \& Performance, 23, 72-85.

Rogers, B. J., \& Bradshaw, M. F. (1994). Is dif-frequency a stimulus for stereoscopic slant? [Abstract.] Investigative Ophthalmology \& Visual Science, 35, 1316.

Schor, C. M., \& Wood, I. (1983). Disparity range for local stereopsis as a function of luminance spatial frequency. Vision Research, 23, 1649-1654.

Schumer, R. A., \& Julesz, B. (1984). Binocular disparity modulation sensitivity to disparities offset from the plane of fixation. Vision Research, 24, 533-542.

SEDGWICK, H. A. (1986). Space perception. In K. R. Boff, L. Kaufman, \& J. P. Thomas (Eds.), Handbook of perception and human performance: Sensory processes (pp. 21.1-21.57). New York: Wiley.

ToYE, R. C. (1986). The effect of viewing position on the perceived layout of space. Perception \& Psychophysics, 40, 85-92.
Wagner, M. (1985). The metric of visual space. Perception \& Psychophysics, 38, 483-495.

WAGNER, M. (2006). The geometries of visual space. London: Erlbaum.

Westheimer, G., \& TAnzman, I. J. (1956). Qualitative depth localization with diplopic images. Journal of the Optical Society of America, 46, 116-117.

Wolfe, U., Maloney, L. T., \& TAM, M. (2005). Distortions of perceived length in the frontoparallel plane: Tests of perspective theories. Perception \& Psychophysics, 67, 967-979.

Woo, G. C. S., \& SillanpaA, V. (1979). Absolute stereoscopic thresholds as measured by crossed and uncrossed disparities. American Journal of Optometry \& Physiological Optics, 56, 350-355.

(Manuscript received August 9, 2006;

revision accepted for publication November $7,2007$. 